\title{
MicroRNA-21 in the pathogenesis of acute kidney injury
}

\author{
Ya-Feng $\mathrm{Li}^{1,2 \bowtie}$, Ying Jing ${ }^{3}$, Jielu Hao ${ }^{4}$, Nathan C Frankfort ${ }^{5}$, Xiaoshuang Zhou ${ }^{1,2}$, Bing Shen ${ }^{6}$, \\ Xinyan Liu ${ }^{1,2}$, Lihua Wang ${ }^{1,2}$, Rongshan $\mathrm{Li}^{2,7}$ \\ ${ }^{1}$ Department of Nephrology and Hemodialysis Center, Second Hospital of Shanxi Medical University, Taiyuan 030001, China \\ 2 Shanxi Renal Disease Research Institution, Taiyuan 030001, China \\ ${ }^{3}$ Department of Blood Purification, General Hospital of Jinan Military Command of PLA, Jinan 250031, China \\ ${ }^{4}$ Nephrology Institute of PLA, Changzheng Hospital of Second Military Medical University, Shanghai 200003, China \\ ${ }^{5}$ Department of Neuroscience and Neurovirology, Temple University School of Medicine, Philadelphia, PA 19140, USA \\ ${ }^{6}$ Department of Urology, Shanghai First People's Hospital, Shanghai 200080, China \\ ${ }^{7}$ Department of Nephrology and Hemodialysis Center, Shanxi Provincial People's Hospital, Taiyuan 030012, China \\ $\triangle$ Correspondence: Dr.yafengli@gmail.com (Y.-F., Li), rongshanli@126.com (R. Li) \\ Received September 13, 2013 Accepted October 7, 2013
}

\section{ABSTRACT}

Acute kidney injury (AKI), associated with significant morbidity and mortality, is widely known to involve epithelial apoptosis, excessive inflammation, and fibrosis in response to ischemia or reperfusion injury, which results in either chronic pathological changes or death. Therefore, it is imperative that investigations are conducted in order to find effective, early diagnoses, and therapeutic targets needed to help prevent and treat AKI. However, the mechanisms modulating the pathogenesis of AKI still remain largely undetermined. MicroRNAs (miRNAs), small noncoding RNA molecules, play an important role in several fundamental biological and pathological processes by a post transcriptional regulatory function of gene expression. MicroRNA-21 (miR-21) is a recently identified, typical miRNA that is functional as a regulator known to be involved in apoptosis as well as inflammatory and fibrotic signaling pathways in AKI. As a result, miR-21 is now considered a novel biomarker when diagnosing and treating AKI. This article reviews the correlative literature and research progress regarding the roles of miR-21 in AKI.

KEYWORDS microRNA, microRNA-21, gene expression, acute kidney injury

\section{INTRODUCTION}

Acute kidney injury (AKI) is a common and serious disease that is costly to treat and often results in death. The incidence of $\mathrm{AKI}$ is on the rise in both high-income and low-income countries. Nearly 600,000 cases of AKI are reported each year in the United States (Rifkin et al., 2012). However, the numbers are more severe in China with AKI affecting approximately one in a thousand individuals per year. Although insight into the causes and mechanisms of AKI is currently increasing, few early diagnostic and therapeutic options exist, which limits the timeframe of recovery to within only a few weeks of occurrence. In recent decades, compelling evidence has illuminated the pathological changes in AKI to include apoptosis, extensive inflammation, fibrosis, and ischemia reperfusion injury (IRI), which induce the expression of many transcriptional regulators and the activation of several signaling pathways.

MicroRNAs (miRNAs) are a class of small noncoding RNA molecules that regulate gene expression by functioning as a post-transcriptional regulatory factor. Consequently, nearly every biological process is estimated to be under the control of miRNAs. It has been recently demonstrated that miRNAs are essential regulators of a number of signaling cascades involved in the pathogenesis of renal disease in vivo and in vitro. Likewise, it has been confirmed that dysfunctional miRNA expression, more specifically the expression of microRNA-21 (miR-21), has played a role in the up-regulation of mechanisms responsible for the progression of AKI. Therefore, using miRNAs as biomarkers as well as other targeted therapeutic strategies could provide early diagnosis and more efficient treatment of AKI. In this review, we summarize our current knowledge about the role of miR-21 and its mechanism that is potentially associated with $\mathrm{AKI}$ pathogenesis. 


\section{MicroRNA AND ITS TARGET MOLECULES}

MiRNAs are a class of noncoding RNA duplex molecules that are approximately 21-23 nucleotides in length, which regulate the stability or the translational efficiency of target mRNAs (Zamore and Haley, 2005). MiRNAs are formed through primary transcription in the nucleus by means of RNA polymerase, also referred to as long primary transcripts (pre-miRNAs), processed into 70- to 100-nucleotide sequences by RNase III Drosha, and then exported into the cytoplasm where they are further processed to generate mature miRNA species of the form of double stranded RNAs (Vasudevan et al., 2007). MiRNAs have been shown to target a number of genes by either enhancing or inhibiting their expression via binding to the 3'-untranslated region (3'UTR) of their target genes. Within a given cell type, both cell-specific and diverse miRNAs can target several mRNAs. As a result, miRNAs exert control over several vital processes of cellular activity and homeostasis including cell growth, proliferation, differentiation, and apoptosis.

\section{MiR-21}

The amount of understanding surrounding the biological functions of many miRNAs including miR-21 has burgeoned in the past decades (Friedman and Jones, 2009; Krichevsky and Gabriely, 2009). However, unlike many other miRNAs, miR-21 is ubiquitously expressed in mammal organ systems such as the heart, spleen, small intestine, and the kidney (Lagos-Quintana et al., 2002). The location of the human miR-21 gene is identified on chromosome 17q23.2, where it overlaps with the protein-coding gene VMP1, also known as TMEM49. In more recent years, there have been many conserved enhancer elements found to positively regulate miR-21 transcription, including binding sites for activation protein 1 (AP-1), which is composed of Fos and Jun family proteins, Ets/PU.1, C/EBPa, NFI, SRF, p53, and STAT3 (Kumarswamy et al., 2011). In addition, several transcriptional suppressors have also been reported, including NFI, C/EBPa, Gf1, and estrogen receptor (Fujita et al., 2008; Velu et al., 2009). MiR-21 expression is also regulated at the post-transcriptional level by signal transducers such as TGF $\beta$, BMP4, and SMADs (Kumarswamy et al., 2011). As such, the controlled or down-regulated expression of miR-21 is tightly associated with several fundamental biological and pathological processes. Accumulating evidence also shows miR-21 plays a vital role in stress response, inflammation, apoptosis, and IRI. Additionally, up-regulation of miR-21 expression has been found to be correlated with multiple kidney diseases such as fibrosis, diabetic nephropathy, renal cell carcinoma (RCC), and AKI (Zhang et al., 2012; Chung et al., 2013; Zhong et al., 2013a). Although most of the regulatory effects of miR-21 on $\mathrm{AKI}$ are still not clear, it is reasonable to predict that many novel functions of miR-21 in this field will be uncovered in the near future.

\section{MiR-21 AND APOPTOSIS}

Considering tubular epithelial cells (TECs) are damaged as a result of $\mathrm{AKI}$, the natural cellular response is programmed death through both necrotic and apoptotic mechanisms (Humphreys et al., 2008). Apoptosis is carried out through both an intrinsic pathway and an extrinsic pathway that ultimately result in the activation of effector apoptotic-specific proteases, which cleave structural proteins, signal transducers, regulators of transcription, repair factors, and many other targets within the cell (Buscaglia and Li, 2011). However, the onset of AKI does not always lead to cell death, rather it is dependently associated with TECs that survive from the insult. Surviving TECs then dedifferentiate and proliferate to reconstitute a functioning nephron (Humphreys et al., 2011). MiR-21 has been reported to promote such proliferation and act as a strong anti-apoptotic factor to inhibit cell death (Chan et al., 2005; Cheng et al., 2010; Godwin et al., 2010). Recent findings have also shown that up-regulation of miR-21 contributed to a reduction in renal cell apoptosis and it also consistently shows protective effects against renal IRI after ischemic preconditioning (IPC) (Xu et al., 2012).

Programmed cell death 4 (PDCD4) was among the earliest confirmed miR-21 targets in RCC, which therefore indicates that miR-21 is associated with growth, apoptosis, and cell cycle progression (Lv et al., 2013). Furthermore, miR-21 overexpression was found to down-regulate promoter activity of PDCD4 by directly targeting the 3'UTR (Lu et al., 2008). While knockdown of miR-21 induced significant up-regulation of PDCD4 as well as tensin homolog (deleted on chromosome 10) and phosphatase, two other proapoptotic target effectors of miR21 , it also resulted in significant down-regulation of phosphorylated protein kinase $B$ and ultimately increased TECs apoptosis (Jia et al., 2013). Moreover, in response to Ras signaling, AP-1, a transcription factor that induces miR-21 expression, was shown to inhibit PDCD4, leading to a decrease in interleukin (IL)-6 and NF-kB induced apoptosis (Talotta et al., 2009; Sheedy et al., 2010). In addition to stimulating apoptosis, NF$\mathrm{KB}$ is also known to induce the expression of miR-21 (Sheedy et al., 2010). However, more is to be learned about the specific mechanisms mediating the overall induction of miR-21 and the role of miR-21 as a negative regulator in the apoptosis of TECs involved in AKI.

\section{MiR-21 AND INFLAMMATION}

IRI-induced AKI is tightly associated with a robust tubulointerstitial inflammation response to hypoxia and the process of reperfusion (Bonventre and Zuk, 2004), both of which not only immediately induce endothelial and tubular cell damage in the initial phase and persistent vasoconstriction in the early phase of AKI, but also subsequently recruit leukocytes leading to the apoptosis and necrosis of endothelial cells and TECs (Rana et al., 2001). The process of renal TEC apoptosis further constitutes a pro-inflammatory stimulus along with a variety of soluble mediators that activate and recruit leukocytes, thereby contributing substantially to the cascade effect (Daemen et al., 1999). A number of potent mediators produced by the injured TECs have been identified as contributors to inflammation, 
including pro-inflammatory cytokines such as IL-6, IL-1 $\beta$, IL-8, tumor necrosis factor (TNF), monocyte chemotactic protein-1 (MCP-1), and transforming growth factor- $\beta 1$ (TGF- $\beta 1$ ) (Akcay et al., 2009). In addition, the extensive inflammation found in renal tissue in response to AKI was initially recognized from Toll-like receptors (TLRs), which activate effector cells via several kinases and NF-KB, while also excreting pro-inflammatory cytokines (Jang and Rabb, 2009). It was found that type I interferon (IFN) signaling downstream of TLR2/TLR4 activation likewise played a key role in the mechanism of I/R-triggered kidney damage (Freitas et al., 2011). Additionally, miR-21 has been found to be highly up-regulated in rat kidneys following tubular injury induced by IRI.

However, in addition to promoting proliferative responses via up-regulation of the survival factor $\mathrm{Bcl}-2$ and down-regulation of the tumor suppressor PDCD4 (Godwin et al., 2010), miR-21 is thought to be involved in inflammation regulation via other signaling pathways. Recent studies suggest that the amount of macrophage infiltration into diabetic kidneys was markedly reduced after treatment with miR-21 plasmids. Similarly, suppression of miR-21 caused a reduction in the pro-inflammatory cytokine TNF- $\alpha$ and MCP-1 (Zhong et al., 2013a). In addition, during a novel study involving the hepatitis $\mathrm{C}$ virus $(\mathrm{HCV})$, researchers observed $\mathrm{HCV}$-mediated activation of miR-21 expression (Chen et al., 2013). Such findings demonstrate that the over-expression of miR-21 targeting MyD88 and IRAK1 results in the activation of certain TLRs that trigger MAPK and NF-KB signaling to produce pro-inflammatory cytokines (Dobrovolskaia et al., 2003; Takeda et al., 2003; Hao et al., 2013). MyD88 consists of a Toll/IL-1 receptor (TIR) domain that mediates TLR signaling, and a death domain (DD) that mediates downstream IL-1 receptor-associated kinase (IRAK)-4 via DD-DD interaction to form MyD88-IRAK-4 complex which recruits IRAK-1 and IRAK-2, thereby bringing their kinase-like domains close together, which results in phosphorylation of IRAKs and their subsequent activation. Phosphorylated IRAK1 or IRAK-2 leaves the complex and interacts with the tumor necrosis factor receptor associated factor 6 (TRAF6). Then the complex signaling cascades of phosphorylation and ubiquitination events are initiated, which lead to phosphorylation of IKKa and MAP kinases, and activation of transcription factors including NF-KB, IRFs, and AP-1 family members and production of inflammatory cytokines (O'Neill and Bowie, 2007; Kawagoe et al., 2008; Carpenter and O'Neill, 2009; Jenkins and Mansell, 2010; Lin et al., 2010).

\section{MiR-21 AND FIBROSIS}

Following severe AKI, the proximal tubule cellular repair process can lead to fibrosis due to cell cycle arrest at the $G_{2} / M$ phase. The disturbed balance between hepatocyte growth factor (HGF) and TGF- $\beta 1$ during the initial stage of AKI, due to increased synthesis of native and foreign HGF in damaged TECs, leads to the generation of pro-fibrotic factors including cytokines, growth factors, and matrix proteins (Yang et al.,
2011). Consequently, AKI can result in proliferation of fibroblasts and excessive deposition of extracellular matrix (Yang et al., 2011; Du et al., 2013b). MiR-21 expression was found to positively correlate with the urine albumin creatine ratio (ACR), tissue inhibitors of metalloproteinase-1 (TIMP1), collagen IV (CollV), and fibronectin (FN), which exacerbate tissue fibrosis; while negatively correlating with the creatine clearance ratio (Ccr) and matrix metalloproteinases-9 (MMP-9) protein, which mitigate the fibrosis process by degrading extracellular matrix components in diabetic nephropathy (Wang et al., 2013). Furthermore, much evidence shows miR-21 regulates the process of renal injury that leads to fibrosis through several signaling pathways. In one study, MiR-21 was found to be significantly increased via the mammalian target of rapamycin (mTOR)-inhibition, which promotes the processing of the miR21 transcript (pri-miR-21) into a premature form (pre-miR-21) (Trindade et al., 2013). Meanwhile, miR-21 Sponge, which quenches endogenous miR-21 levels, attenuated phosphorylation of endogenous inhibitors of mTORC1 by inhibiting TGF $\beta$-stimulated phosphorylation of Akt kinase (Dey et al., 2012). Another study conducted in vivo identified that miR-21 targeting of Smad7 was involved in TGF- $\beta$ and NF-KB signaling pathways. Overexpression of miR-21 in kidney cells was shown to enhance the production of fibrotic and inflammatory markers, while knockdown of miR-21 produced opposite results (Zhong et al., 2013b). Likewise, a strong up-regulation of miR-21 was observed in the kidneys of mice suffering from unilateral ureteral obstruction where primary kidney fibroblasts were induced upon TGF $\beta$. Such results indicate that ectopic expression of miR-21 in primary kidney fibroblasts is sufficient to promote myofibroblast differentiation (Glowacki et al., 2013). These findings imply that miR-21 plays a complicated role in the regulation of the fibrotic repair process after AKI.

\section{MiR-21 AND ISCHEMIA REPERFUSION INJURY}

Clinical studies have shown that renal IRI is a major cause of AKI (Lameire and Vanholder, 2001; Lameire et al., 2006). Ischemia markedly initiates injury of both the renal tubular epithelium and renal microvasculature, which is exacerbated upon reperfusion by an increase in oxidative stress, inflammation, apoptosis, and necrosis (Bonventre and Weinberg, 2003; Devarajan, 2006). MiR-21 down-regulated apoptosis and necrosis of renal TECs, which promoted cellular proliferation and played a protective role in the response to stress and inflammation in renal IRI (Lindsay, 2008; Thum et al., 2008a; Godwin et al., 2010). As shown in a previous animal study, knockdown of miR-21 increased cell death. However, a conflicting result was also noted in vitro: over-expression of miR-21 did not prevent cell death following simulated ischemia (Godwin et al., 2010). These results indicated the role of miR-21 is a two sided sword in renal IRI: neither under- nor over-expression led to adverse consequences (Fig. 1). Under-expression of miR-21 results in the increase of cell death since miR-21 acts as an anti-apoptosis agent. In contrast, miR-21 over-expression can 


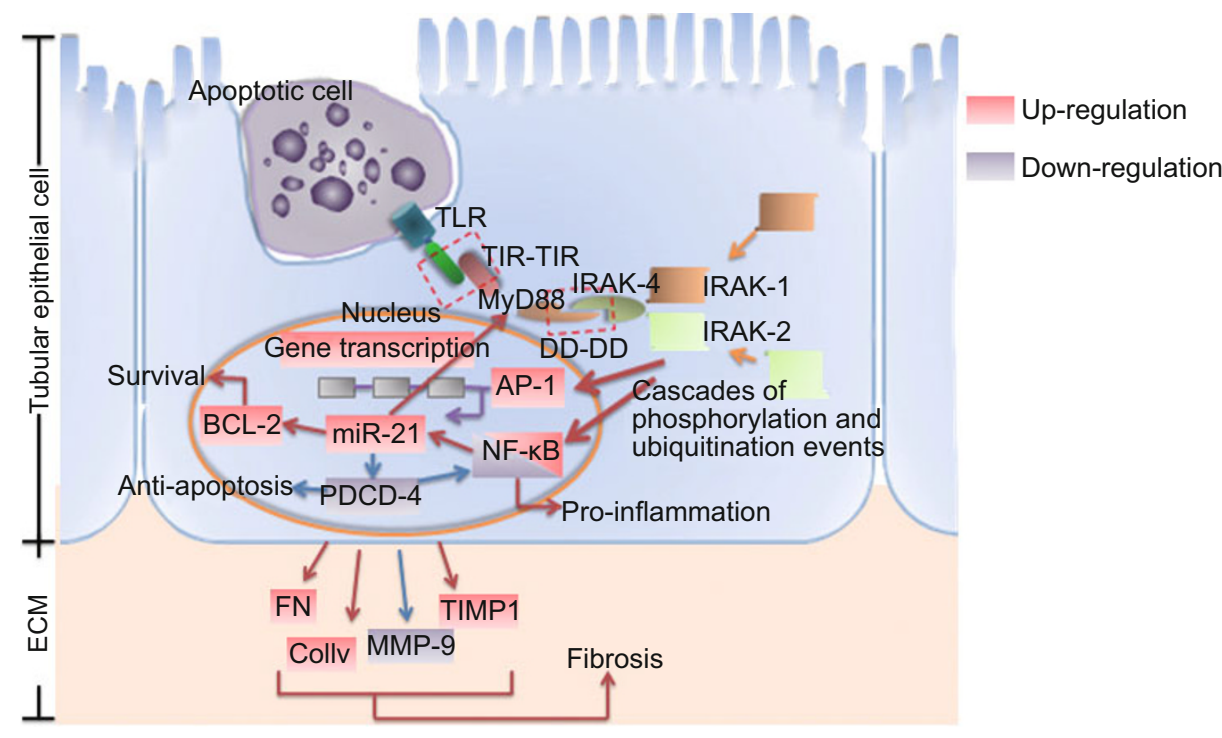

Figure 1. Two-side sword effect of miR-21 in AKI. On one hand, in the initial stage of AKI, miR-21 up-regulation switches on a defense mechanism against the stimulation of apoptosis recognized by TLRs, which down-regulates PDCD4 by directly targeting the 3'UTR, as well as up-regulates survival factor Bcl-2, which acts as a strong anti-apoptotic factor to inhibit cell death. Meanwhile, inhibition of PDCD4 leads to a decrease in NF-KB induced apoptosis. However, on the other hand, in the end stage of AKI, over-expression of miR-21 targeting MyD88 and IRAK1 results in the continuous activation of certain TLRs to amplify cascades of phosphorylation and ubiquitination events to trigger NF-KB to produce proinflammatory cytokines. Moreover, excessive up-regulation of miR-21 positively correlates with TIMP1, CollV, and FN, while negatively correlating with MMP-9, which exacerbates tissue fibrosis by intensifying cell death and tissue injury.

activate several signaling pathways leading to inflammation and fibrosis. Therefore, there should be a precise dynamic adjustment mechanism in physiological condition. After suffering ischemia assault, the balance will be destroyed. Expression of miR-21 may be down-regulated in the initial stage of IRI, and up-regulated as a self-defense response and thereby performing an anti-apoptosis effect in spite of the massive death of TECs in the subsequent stage. However, over-expression in the end stage leads to the production of pro-inflammatory and pro-fibrotic factors. Another recent study supported this showing that 15-min renal IPC significantly increased the expression of miR-21 by $4 \mathrm{~h}$ and substantially attenuated ischemia-reperfusion injury that was induced 4 days later. On the other hand, knockdown of miR-21 resulted in significant up-regulation of PCDP4 and a substantial increase in tubular cell apoptosis. The same study also showed that miR-21 performed a renal protective effect by further delaying IPC against subsequent renal IRI (Xu et al. 2012). Therefore, up-regulation of miR-21 during a short period of ischemia followed by reperfusion can activate endogenous defense mechanisms that protect against a subsequent, sustained ischemic insult; however, imbalanced expression may contribute to a serious irreversible outcome.

\section{MIR-21 AS A DIAGNOSTIC BIOMARKER AND THERAPEUTIC TARGET}

MiRNAs are rapidly emerging as early diagnostic biomarkers in human diseases due to the noted release of miRNAs from specific tissue into body fluids during the onset of certain negative physiological and pathological conditions in the body (Laterza et al. 2009). MiRNAs were found to be differentially regulated following IRI, which led to problems such as apoptosis (Ren et al. 2009), fibrosis (Thum et al. 2008), epithelial mesenchymal transition (Gregory et al. 2008), and TLR signaling (Taganov et al. 2006). Expression of miR-21 was found to be robustly up-regulated following ischemia in TECs (Saikumar et al. 2012). Furthermore, miR-21 may play a role in fine-tuning the cellular responses to injury in the kidney (Godwin et al. 2010). Another recent study estimated that the levels of urinary and plasma miR-21 were related to severe AKI and other complications following cardiac surgery. In addition, the predictive power of miR-21 for the progression of AKI was comparable to previously reported biomarkers such as the creatinine ratio, urine neutrophil gelatinase-associated lipocalin (NGAL), urine IL-18, and plasma NGAL (Koyner et al. 2012; Du et al. 2013). With methodological advances, artificial modification of miR-21 is very likely to be employed not only in the early diagnosis but also in the prevention and treatment of AKI in the near future.

\section{CONCLUSIONS}

AKI is a pathological condition characterized by epithelial apoptosis, recruit of leukocytes, and activation of proinflammatory cytokines at the early stage of injury, secretion of pro-fibrotic factors, proliferation of fibroblasts, and excessive deposition of extracellular matrix at the terminal stage of injury. MiR-21 is a 
typical miRNA that functions as a regulator involved in several fundamental biological and pathologic processes. Previous studies confirmed the extensive involvement of miR-21 in apoptosis and both inflammatory and fibrotic signaling pathways; however, it was found that miR-21 might play an opposite role in AKI. On one hand, up-regulation of miR-21 acts as a defense mechanism to decrease cell death. On the other hand, over-expression of miR-21, stimulated by massive cell death, results in severe inflammation and fibrosis. Therefore, there should be a precise dynamic adjustment to miR-21 in physiological condition. In addition, miR-21 is identified as a novel biomarker for early diagnosis and a potential therapeutic target in preventing and treating AKI. However, the mechanisms mediating the induction of miR-21 following AKI have not been experimentally validated. Furthermore, it should also be noted that large-scale studies are required to evaluate the clinical values of miR-21 as predictive markers in AKI.

\section{ABBREVIATIONS}

AKI, acute kidney injury; AP-1, activation protein 1; HGF, hepatocyte growth factor; IPC, ischemic preconditioning; IRI, ischemia reperfusion injury; PDCD4, programmed cell death 4; RCC, renal cell carcinoma; TECs, tubular epithelial cells; TLRs, Toll-like receptors

\section{COMPLIANCE WITH ETHICS GUIDELINES}

Ya-Feng Li, Ying Jing, Jielu Hao, Nathan Frankfort, Xiaoshuang Zhou, Bing Shen, Xinyan Liu, Lihua Wang and Rongshan Li declare that they have no conflict of interest.

This article does not contain any studies with human or animal subjects performed by the any of the authors.

\section{REFERENCES}

Akcay, A., Nguyen, Q., and Edelstein, C.L. (2009). Mediators of inflammation in acute kidney injury. Mediators Inflamm 2009, 137072.

Bonventre, J.V., and Weinberg, J.M. (2003). Recent advances in the pathophysiology of ischemic acute renal failure. J Am Soc Nephrol 14, 2199-2210.

Bonventre, J.V., and Zuk, A. (2004). Ischemic acute renal failure: an inflammatory disease? Kidney Int 66, 480-485.

Buscaglia, L.E.B., and Li, Y. (2011). Apoptosis and the target genes of microRNA-21. Chin J Cancer 30, 371-380.

Carpenter, S., and O'Neill, L. (2009). Recent insights into the structure of Toll-like receptors and post-translational modifications of their associated signalling proteins. Biochem J 422, 1-10.

Chan, J.A., Krichevsky, A.M., and Kosik, K.S. (2005). MicroRNA-21 is an antiapoptotic factor in human glioblastoma cells. Cancer Res 65, 6029-6033.

Chen, Y., Chen, J., Wang, H., Shi, J., Wu, K., Liu, S., Liu, Y., and Wu, J. (2013). HCV-induced miR-21 contributes to evasion of host immune system by targeting MyD88 and IRAK1. PLoS Pathog 9, e1003248.

Cheng, Y., Zhu., P., Yang, J., Liu, X., Dong, S., Wang, X., Chun, B., Zhuang, J., and Zhang, C. (2010). Ischaemic preconditioning-regulated miR-21 protects heart against ischaemia/reperfusion injury via anti-apoptosis through its target PDCD4. Cardiovasc Res 87,
431-439.

Chung, A.C.K., Dong, Y., Yang, W., Zhong, X., Li, R., and Lan, H.Y. (2013). Smad7 suppresses renal fibrosis via altering expression of TGF- miR-21 protects hmicroRNAs. Mol Ther 21, 388-398.

Daemen, M.A., Veer, C.V.t., Denecker, G., Heemskerk, V.H., Wolfs, T.G., Clauss, M., Vandenabeele, P., and Buurman, W.A. (1999). Inhibition of apoptosis induced by ischemia-reperfusion prevents inflammation. J Clin Invest 104, 541-549.

Devarajan, P. (2006). Update on mechanisms of ischemic acute kidney injury. J Am Soc Nephrol 17, 1503-1520.

Dey, N., Ghosh-Choudhury, N., Kasinath, B.S., and Choudhury, G.G. (2012). TGF Am Soc Nephrol 17, 1503 and Buurman, W.A. (1999). Inhibition of apoptosis induced by ischemia-reperfusion prevents inflammation. J Clin 7, e42316.

Dobrovolskaia, M.A., Medvedev, A.E., Thomas, K.E., Cuesta, N., Toshchakov, V., Ren, T., Cody, M.J., Michalek, S.M., Rice, N.R., and Vogel, S.N. (2003). Induction of in vitro reprogramming by Toll-like receptor (TLR)2 and TLR4 agonists in murine macrophages: effects of TLR «homotolerance» versus «heterotolerance» on NF-kappa B signaling pathway components. J Immunol 170, 508-519.

Du, J., Cao, X., Zou, L., Chen, Y., Guo, J., Chen, Z., Hu, S., and Zheng, Z. (2013a). MicroRNA-21 and risk of severe acute kidney injury and poor outcomes after adult cardiac surgery. PLoS One 8, e63390.

Du, T., Zou, X., Cheng, J., Wu, S., Zhong, L., Ju, G., Zhu, J., Liu, G., Zhu, Y., and Xia, S. (2013b). Human Wharton's jelly-derived mesenchymal stromal cells reduce renal fibrosis through induction of native and foreign hepatocyte growth factor synthesis in injured tubular epithelial cells. Stem Cell Res Ther 4, 59. (In Press).

Freitas, M.C.S., Uchida, Y., Lassman, C., Danovitch, G.M., Busuttil, R.W., and Kupiec-weglinski, J.W. (2011). Type I interferon pathway mediates renal ischemia/reperfusion injury. Transplantation 92, 131-138.

Friedman, J.M., and Jones, P.A. (2009). MicroRNAs: critical mediators of differentiation, development and disease. Swiss Med Wkly 139, 466-472.

Fujita, S., Ito, T., Mizutani, T., Minoguchi, S., Yamamichi, N., Sakurai, K., and Iba, H. (2008). miR-21 Gene expression triggered by AP-1 is sustained through a double-negative feedback mechanism. J Mol Biol 378, 492-504.

Glowacki, F., Savary, G., Gnemmi, V., Buob, D., Hauwaert, C.V.D., Loguidice, J.-m., Bouy (2008). miR-21 Gene expression triggered b, et al. (2013). Increased circulating miR-21 levels are associated with kidney fibrosis. PLoS One 8, e58014.

Godwin, J.G., Ge, X., Stephan, K., Jurisch, A., Tullius, S.G., and laComini, J. (2010). Identification of a microRNA signature of renal ischemia reperfusion injury. Proc Natl Acad Sci U S A 107, 1433914344.

Gregory, P.A., Bracken, C.P., Bert, A.G., and Goodall, G.J. (2008). MicroRNAs as regulators of epithelial-mesenchymal transition. Cell Cycle 7, 3112-3118.

Hao, J.L., Li, Y.F., and Li, R.S. (2013). A novel mechanism of NALP3 inducing ischemia reperfusion injury by activating MAPK pathway in acute renal failure. Medical hypotheses 80, 463-465.

Humphreys, B.D., Czerniak, S., Dirocco, D.P., Hasnain, W., Cheema, R., and Bonventre, J.V. (2011). Repair of injured proximal tubule 
does not involve specialized progenitors. Proc Natl Acad Sci U S A 108.

Humphreys, B.D., Valerius, M.T., Kobayashi, A., Mugford, J.W., Soeung, S., Duffield, J.S., Mcmahon, A.P., and Bonventre, J.V. (2008). Intrinsic epithelial cells repair the kidney after injury. Cell Stem Cell 2, 284-291.

Jang, H.R., and Rabb, H. (2009). The innate immune response in ischemic acute kidney injury. Clin Immunol 130, 41-50.

Jenkins, K., and Mansell, A. (2010). TIR-containing adaptors in Toll-like receptor signalling. Cytokine 49, 237-244.

Jia, P., Teng, J., Zou, J., Fang, Y., Zhang, X., Bosnjak, Z.J., Liang, M., and Ding, X. (2013). miR-21 Contributes to Xenon-conferred Amelioration of Renal Ischemia-Reperfusion Injury in Mice. Anesthesiology 119, 621-630.

Kawagoe, T., Sato, S., Matsushita, K., H, H.K., Matsui, K., Kumagai, Y., Saitoh, T., Kawai, T., Takeuchi, O., and Akira, S. (2008). Sequential control of Toll-like receptor-dependent responses by IRAK1 and IRAK2. Nat Immunol 9, 684-691.

Koyner, J.L., Garg, A.X., Coca, S.G., Sint, K., Thiessen-Philbrook, H., Patel, U.D., Shlipak, M.G., and Parikh, C.R. (2012). Biomarkers predict progression of acute kidney injury after cardiac surgery. $\mathrm{J}$ Am Soc Nephrol 23, 905-914.

Krichevsky, A.M., and Gabriely, G. (2009). miR-21: a small multi-faceted RNA. J Cell Mol Med 13, 39-53.

Kumarswamy, R., Volkmann, I., and Thum, T. (2011). Regulation and function of miRNA-21 in health and disease. RNA Biol 8, 706-713.

Lagos-Quintana, M., Rauhut, R., Yalcin, A., Meyer, J., Lendeckel, W., and Tuschl, T. (2002). Identification of tissue-specific microRNAs from mouse. Curr Biol 12, 735-739.

Lameire, N., Biesen, W.V., and Vanholder, R. (2006). The changing epidemiology of acute renal failure. Nat Clin Pract Nephrol 2, 364377.

Lameire, N., and Vanholder, R. (2001). Pathophysiologic features and prevention of human and experimental acute tubular necrosis. $\mathrm{J}$ Am Soc Nephrol 12 Suppl 17, S20-32.

Laterza, O.F., Lim, L., Garrett-Engele, P.W., Vlasakova, K., Muniappa, N., Tanaka, W.K., Johnson, J.M., Sina, J.F., Fare, T.L., Sistare, F.D., et al. (2009). Plasma MicroRNAs as sensitive and specific biomarkers of tissue injury. Clin Chem 55, 1977-1983.

Lin, S., Lo, Y., and Wu, H. (2010). Helical assembly in the MyD88IRAK4-IRAK2 complex in TLR/IL-1R signalling. Nature 465, 885890.

Lindsay, M. (2008). microRNAs and the immune response. Trends Immunol 29, 343-351.

Lu, Z., Liu, M., Stribinskis, V., Klinge, C.M., Ramos, K.S., Colburn, N.H., and Li, Y. (2008). MicroRNA-21 promotes cell transformation by targeting the programmed cell death 4 gene. Oncogene 27 , 4373-4379.

Lv, L., Huang, F., Mao, H., Li, M., Li, X., Yang, M., and Yu, X. (2013). MicroRNA-21 is overexpressed in renal cell carcinoma. Int $\mathrm{J}$ Biol Markers 28, e201-207.

O'Neill, L., and Bowie, A. (2007). The family of five: TIR-domain-containing adaptors in Toll-like receptor signalling. Nat Rev Immunol 7, 353-364.

Rana, A., Sathyanarayana, P., and Lieberthal, W. (2001). Role of apoptosis of renal tubular cells in acute renal failure: therapeutic implications. Apoptosis 6, 83-102.
Ren, X.-P., Wu, J., Wang, X., Sartor, M.A., Qian, J., Jones, K., Nicolaou, P., Pritchard, T.J., and Fan, G.-C. (2009). MicroRNA-320 is involved in the regulation of cardiac ischemia/reperfusion injury by targeting heat-shock protein 20. Circulation 119, 2357-2366.

Rifkin, D.E., Coca, S.G., and Kalantar-Zadeh, K. (2012). Does AKI truly lead to CKD? J Am Soc Nephrol 23, 979-984.

Saikumar, J., Hoffmann, D., Kim, T.-m., Gonzalez, V.R., Zhang, Q., Goering, P.L., Brown, R.P., Bijol, V., Park, P., Waikar, S.S., et al. (2012). Expression, circulation, and excretion profile of microRNA-21, -155 , and -18 a following acute kidney injury. Toxicol Sci 129, 256-267.

Sheedy, F.J., Palsson-mcdermott, E., Hennessy, E.J., Martin, C., O'leary, J.J., Ruan, Q., Johnson, D.S., Chen, Y., and O'neill, L.A.J. (2010). Negative regulation of TLR4 via targeting of the proinflammatory tumor suppressor PDCD4 by the microRNA miR-21. Nat Immunol 11, 141-147.

Taganov, K.D., Boldin, M.P., Chang, K.-J., and Baltimore, D. (2006). NF-kappaB-dependent induction of microRNA miR-146, an inhibitor targeted to signaling proteins of innate immune responses. Proc Natl Acad Sci U S A 103, 12481-12486.

Takeda, K., Kaisho, T., and Akira, S. (2003). Toll-like receptors. Annu Rev Immunol 21, 335-376.

Talotta, F., Cimmino, A., Matarazzo, M.R., Casalino, L., Vita, G.D., D'esposito, M., Lauro, R.D., and Verde, P. (2009). An autoregulatory loop mediated by miR-21 and PDCD4 controls the AP-1 activity in RAS transformation. Oncogene 28, 73-84.

Thum, T., Catalucci, D., and Bauersachs, J. (2008a). MicroRNAs: novel regulators in cardiac development and disease. Cardiovasc Res 79, 562-570.

Thum, T., Gross, C., Fiedler, J., Fischer, T., Kissler, S., Bussen, M., Galuppo, P., Just, S., Rottbauer, W., Frantz, S., et al. (2008b). MicroRNA-21 contributes to myocardial disease by stimulating MAP kinase signalling in fibroblasts. Nature 456, 980-984.

Trindade, A.J., Medvetz, D.A., Neuman, N.A., Myachina, F., Yu, J., Priolo, C., and Henske, E.P. (2013). MicroRNA-21 is induced by rapamycin in a model of tuberous sclerosis (TSC) and lymphangioleiomyomatosis (LAM). PLoS One 8, e60014.

Vasudevan, S., Tong, Y., and Steitz, J. (2007). Switching from repression to activation: microRNAs can up-regulate translation. Science 318, 1931-1934.

Velu, C.S., Baktula, A.M., and Grimes, H.L. (2009). Gfi1 regulates miR-21 and miR-196b to control myelopoiesis. Blood 113, 47204728.

Wang, J., Gao, Y., Ma, M., Li, M., Zou, D., Yang, J., Zhu, Z., and Zhao, X. (2013). Effect of miR-21 on Renal Fibrosis by Regulating MMP9 and TIMP1 in kk-ay Diabetic Nephropathy Mice. Cell Biochem Biophys. (In Press)

Xu, X., Kriegel, A.J., Liu, Y., Usa, K., Mladinov, D., Liu, H., Fang, Y., Ding, X., and Liang, M. (2012). Delayed ischemic preconditioning contributes to renal protection by upregulation of miR-21. Kidney Int $82,1167-1175$

Yang, L., Humphreys, B.D., and Bonventre, J.V. (2011). Pathophysiology of acute kidney injury to chronic kidney disease: maladaptive repair. Contrib Nephrol 174, 149-155.

Zamore, P., and Haley, B. (2005). Ribo-gnome: the big world of small RNAs. Science 309, 1519-1524.

Zhang, H., Guo, Y., Shang, C., Song, Y., and Wu, B. (2012). miR-21 
downregulated TCF21 to inhibit KISS1 in renal cancer. Urology 80 , 1298-1302.

Zhong, X., Chung, A.C.K., Chen, H.Y., Dong, Y., Meng, X.M., Li, R., Yang, W., Hou, F.F., and Lan, H.Y. (2013a). miR-21 is a key therapeutic target for renal injury in a mouse model of type 2 diabetes.
Diabetologia 56, 663-674.

Zhong, X., Chung, A.C.K., Chen, H.Y., Dong, Y., Meng, X.M., Li, R., Yang, W., Hou, F.F., and Lan, H.Y. (2013b). miR-21 is a key therapeutic target for renal injury in a mouse model of type 2 diabetes. Diabetologia 56, 663-674. 